\title{
ORIGINAL
}

\section{LA CALIDAD DE VIDA RELACIONADA CON LA SALUD COMO FACTOR EXPLICATIVO DE LA UTILIZACIÓN DE LA CONSULTA DE MEDICINA DE FAMILIA: UN ESTUDIO BAJO EL MODELO CONDUCTUAL (*)}

\author{
Jesús Martín-Fernández (1), Tomás Gómez-Gascón (2), Mª Isabel del Cura-González (3), Nuria \\ Tomás-García (1), Concepción Vargas-Machuca (2) y Gemma Rodríguez-Martínez (4) \\ (1) Centro de salud San Martín de Valdeiglesias. Área 8 de AP. Servicio Madrileño de Salud. \\ (2) Centro de salud Puerta Bonita II. Área 11 de AP. Servicio Madrileño de Salud. \\ (3) Unidad de Docencia e Investigación. Área 9 de AP. Servicio Madrileño de Salud. Universidad Rey Juan Carlos I. \\ (4) Centro de salud Condes de Barcelona. Área 6 de AP. Servicio Madrileño de Salud.
}

\section{RESUMEN}

Fundamento: La utilización de servicios sanitarios se ha estudiado desde varios modelos conceptuales. El objetivo de este trabajo es valorar la influencia de la calidad de vida relacionada con la salud (CVRS) en la variabilidad de la utilización de la consulta de atención primaria bajo el «modelo conductual».

Métodos: Estudio transversal analítico. Se entrevistó a 451 sujetos de 6 centros de salud en la Comunidad de Madrid. Se recogieron características relacionadas con factores ambientales e individuales (predisponentes, facilitadores y de necesidad) y la CVRS (Euroqol-5D). La utilización se recogió de la historia clínica informatizada.

Resultados: Hubo una mediana de 13 visitas/año (rango intercuarti 7-20). La CVRS explicaba un 5,2\% de la variabilidad en la utilización, vivir en barrios de rentas altas un $2,4 \%$, la edad un $7,4 \%$, la renta individual un $1,0 \%$ y los factores relacionados con «necesidad» (enfermedades crónicas e ingresos hospitalarios) explicaban un $4,1 \%$ de variabilidad adicional. Cada cambio de tercil de la CVRS se asoció con una disminución del $13,8 \%$ en las consultas/año. La residencia en zonas de renta alta y cada aumento de 1000 de renta individual disminuían la utilización un 17,5\% y un $9,5 \%$ respectivamente. La edad se asociaba con un aumento de un $8,0 \%$ de visitas/año por década. El sufrir enfermedades crónicas o ingresos hospitalarios suponía aumentar un $51,9 \%$ y un $26,5 \%$ las visitas anuales.

Conclusiones: La CVRS se asocia de manera independiente con la variabilidad en la utilización de la consulta del médico de familia, una vez ajustado el efecto de la necesidad, como propone el «modelo conductual».

Palabras clave: Necesidades y Demandas de Servicios de Salud. Mala utilización de los servicios de salud. Atención Primaria de Salud. Calidad de Vida. Factores Socioeconómicos.

\section{ABSTRACT \\ Quality of Life Related to Health as a Factor Explaining the Use of Family Medical Consultation: A Study on the Behavioral Model}

Background: Health services utilization has been studied under several conceptual models. This study is aimed to assess the influence of Health Related Quality of Life (HRQL) on the variability of the primary care consultation utilization under the «behavioral model»

Methods: A cross-sectional study. Interviews were conducted with 451 subjects at six health centers in the Community of Madrid. Environmental and individual characteristics (predisposing, enabling and need factors) and HRQL (EuroQol-5D) were collected. Annual visits were retrieved from the computerized clinical history.

Results: Median utilization was 13 visits/year (interquartile range 7 20). HRQL explained $5,2 \%$ of utilization variability, living in high-income areas an additional $2.4 \%$, age a $7.4 \%$, incomes a $1.0 \%$ and «need factors» (chronic illnesses or a hospital admission in the last year) explained an additional $4.1 \%$ of the variability. Each increase of the tercile in the perception of HRQL was associated with a decrease of $13,8 \%$ of the number of visits/year. Living in high-income areas, and each increase of 1000 in the incomes decreased the percentage of annual visits $17.5 \%$ and $9.5 \%$ respectively. Age is associated with an increase of $8.0 \%$ of annual visits per decade. Suffering from chronic illnesses or a hospital admission in the last year increased the average number of visits/year to 51.9 and $26.5 \%$.

Conclusions: HRQL is independently associated with the variability of the demand for the family physician after adjusting by « health need» factors as the «behavioral model» proposes.

Key words: Health Services Needs and Demand. Overutilization of Health Services. Primary Health Care. Quality of life. Socioeconomic factors.

(*) Los datos necesarios para la realización de este estudio se recogieron gracias a la beca concedida por el Fondo de Investigación Sanitaria Proyecto $n^{\circ} 070514$, Plan Nacional de Investigación Científica, Desarrollo e Innovación Tecnológica e Instituto de Salud Carlos III.

Ninguno de los autores declara conflictos de intereses al elaborar este trabajo. 


\section{INTRODUCCIÓN}

La planificación de los servicios sanitarios está íntimamente ligada a sus condiciones de utilización. Se ha descrito que apenas un tercio de los pacientes originan el $80 \%$ de las consultas de los médicos de familia ${ }^{1}$, lo que hace especialmente interesante comprender este fenómeno.

Existen varios tipos de modelos teóricos que pretenden conceptualizar la utilización de servicios sanitarios. De entre ellos destaca por su carácter integrador, por su consistencia y por su evolución el denominado «modelo conductual» («behavioral model») propuesto inicialmente por Andersen. Este modelo en su desarrollo ha ido incorporando elementos de teorías alternativas, que eran críticas con sus planteamientos, haciendo notar que no daba el suficiente peso a los factores organizativos, o a las creencias en salud (como proponía Rosenstock en su modelo psicosocial), o a la extensión y calidad de la red social. En su formulación revisada el «modelo conductual» propone la existencia de unos determinantes primarios (características ambientales, y de los individuos) que producen una conducta en salud (hábitos en salud y uso del sistema sanitario), que a su vez producen unos resultados en salud (percepción del estado de salud, un estado de salud evaluable y una satisfacción en el usuario) $)^{2}$. Los determinantes primarios, tanto ambientales como individuales se pueden agrupar en factores predisponentes, facilitadores y de «necesidad $»^{3}$.

El papel de los determinantes primarios ambientales, entre los que se pueden incluir las características del proveedor, del sistema de atención o el entorno físico están ampliamente estudiadas ${ }^{4,5}$. Las características de los individuos pueden agruparse en unos factores predisponentes como la ocupación, la estructura social o la educación; otros facilitadores como el nivel de renta, la existencia de aseguramiento o la accesibilidad de los servicios o los tiempos de espera; y la «necesidad», como principal elemento generador de demanda sanitaria, que a su vez tiene un componente objetivable y otro de percepción.

Entre los factores predisponentes individuales para el uso de los recursos sanitarios, se conoce la asociación entre los factores psicosociales y la desestructuración familiar con un mayor consumo de recursos ${ }^{6,7}$, y una relación inversa entre tamaño familiar y número de consultas en $\mathrm{AP}^{8}$.

De los factores facilitadores, la renta es el principal limitador del acceso a los servicios sanitarios desde una perspectiva global, pero no en los países con sistemas nacionales de salud $^{9}$. En estos, cuando no existe un pago directo a la hora de acceder al servicio, parece haber más uso cuanto menor es la renta para los servicios en atención primaria (una «inequidad pro-pobres») $)^{10,11}$, y una relación menos definida en otros niveles asistencia$\operatorname{les}^{12}$. En estos entornos la accesibilidad al servicio, puede ser el limitante del uso ${ }^{13,14}$.

La influencia de la necesidad en salud sobre la demanda está bien establecida para medidas objetivables como la morbili$\operatorname{dad}^{15,16}$, o la incapacidad funcional ${ }^{17}$.

El estudio de los sistemas de salud con este modelo ha permitido detectar inequidades en el acceso a la atención sanitaria ${ }^{3,18}$, que el propio Andersen definía como una excesiva variabilidad en el uso de servicios ligada a las diferencias en factores ambientales como la estructura social o las creencias en salud, o en factores facilitadores individuales como la renta.

En la evolución del «modelo conductual» se propone la «percepción del estado de salud» como un resultado del contacto con el sistema sanitario que, a su vez actúa sobre las características individuales que generan demanda, cerrando un círculo de interacción. La percepción del estado de salud puede asimilarse al constructo «calidad de vida relacionada con la salud» (CVRS). 
La CVRS parece ser más determinante del uso de servicios sanitarios en el sector privado que en el público ${ }^{19}$. Fuera de sistemas nacionales de salud, la CVRS ha mostrado, junto a la morbilidad, su capacidad para predecir el consumo de recursos en $\mathrm{AP}^{20}$. Dentro de sistemas públicos, una peor percepción de la CVRS se ha mostrado como predictora de un mayor uso de la consulta del médico de familia en determinadas patologías crónicas como la osteomuscular ${ }^{21}$, o la obesidad ${ }^{22}$, pero por sí sola, solo es una débil predictora del sobreuso de recursos ${ }^{23}$.

En nuestro entorno se ha estudiado la utilización de servicios en AP de manera continuada en el tiempo, pero no se ha incidido en la importancia de la CVRS como factor explicativo de la utilización de servicios, dentro de un marco teórico sólido como es el modelo propuesto por Andersen.

En este trabajo se pretende evaluar la capacidad explicativa de la CVRS sobre las diferencias en la utilización de la consulta de medicina de familia en un sistema público de salud y su peso relativo frente a determinados factores predisponentes o facilitadores y frente a la necesidad objetivada como morbilidad.

\section{SUJETOS Y MÉTODOS}

Diseño y sujetos. Se diseñó un estudio transversal analítico para estudiar las variables explicativas de las diferencias en el uso de la consulta del médico de familia, bajo el «modelo conductual».

Los datos se obtuvieron en el contexto de un estudio de evaluación de servicios. Se entrevistó a sujetos que acababan de asistir a una consulta de medicina de familia. Se escogieron por conveniencia 6 centros de salud, 4 urbanos y 2 rurales, que pertenecían a zonas en los terciles alto ( 3 centros) y bajo (3 centros) de la distribución de rentas de la Comunidad de Madrid. Dentro de cada cen- tro las personas fueron seleccionadas de manera aleatoria de los listados de citas, por muestreo sistemático, con remplazamiento en el caso de no querer ser incluidos en el estudio. Todos los pacientes incluidos dieron su consentimiento a participar.

Variables. La variable de resultado fue el número de visitas realizado al médico de familia en el último año y se extraía de la historia clínica informatizada.

La variable de estudio fue la calidad de vida relacionada con la salud (CVRS). Se mensuró utilizando el cuestionario EuroQol5D. Se transformaron las respuestas en utilidades y se dividió su distribución en tres terciles. El tercil superior corresponde a una mejor percepción de la CVRS y el inferior a una CVRS percibida inferior.

Entre las variables de ajuste se recogieron aquellas relacionadas con el «factor ambiental» y con características individuales. Estas últimas se englobaron en factores predisponentes, facilitadores y la necesidad en salud.

Las variables incluidas en los llamados «factores ambientales» fueron el entorno rural o urbano y la renta media de la zona (distribuida en terciles).

Entre las características individuales, además de las características sociodemográficas, como factores predisponentes se incluyeron el máximo nivel de estudios completado, y la ocupación (clases I, la ocupación más cualificada, a V, la menos cualificada) ${ }^{24}$.

Como factores facilitadores se recogieron la existencia o no de otro aseguramiento, la accesibilidad al servicio y la renta individual. La accesibilidad al servicio se estudió mediante el tiempo necesario para obtener cita (desde el mismo día hasta más de tres días) y el tiempo de espera desde la hora de cita hasta la consulta (desde nada a más de una hora). La renta individual se calculó sumando todos los ingresos de la unidad 
familiar y ponderando por el tamaño familiar.

Para valorar la «necesidad objetivable» se constató la existencia de patologías crónicas (definidas como circunstancias de salud que hubiesen requerido atención por más de seis meses) y la existencia o no de ingresos hospitalarios en el último año. Estas dos variables eran comunicadas por el propio médico de familia, tras consultar la historia clínica.

\section{Análisis}

Se estudió la distribución de la utilización y se denominó utilizador frecuente (UF) a aquella persona que había realizado un número de visitas anuales en el tercil superior. Se hizo un estudio descriptivo de las características individuales de los sujetos y se evaluaron las relaciones entre estas y el hecho de pertenecer a la categoría UF.

Se construyó un modelo explicativo en el que la variable dependiente fue el logaritmo neperiano (ln) del número de visitas/año. La variable explicativa principal fue la CVRS representada por la «utilidad» en el EuroQol-5D, y como variables de ajuste se usaron todas aquellas que se asociaban a la utilización individualmente $(\mathrm{si} p<0,10)$. Se introdujeron por bloques, primero la CVRS, luego las variables relacionadas con el ambiente, después las relacionadas con factores individuales predisponentes, facilitadores y por último las relativas a «necesidad». Se seleccionó el modelo con mayor capacidad explicativa con un menor número de variables (principio de parsimonia).

Posteriormente, para estudiar el mismo fenómeno, de forma complementaria, se elaboró un modelo logístico cuya variable dependiente fue la pertenencia o no a la categoría UF. Se evaluó la capacidad que tenía la CVRS para clasificar a un sujeto como UF, ajustando por el resto de variables. Se evaluó también la capacidad de clasificación del modelo.

Por la forma de selección de los sujetos, los datos podían tener estructura jerárquica, pero no había suficientes unidades de análisis para llevar a cabo un análisis mutinivel. Por ello, y en previsión de la existencia de heterocedasticidad, la construcción de los modelos se hizo con estimadores robustos, que permiten estimar coeficientes de regresión consistentes en presencia de una heterocedasticidad no conocida.

El tamaño de la muestra fue calculado para el conjunto del estudio de evaluación de servicios y se estimó en 450 sujetos. Este tamaño permitía construir un modelo inicial en el que se incorporasen todas las posibles variables explicativas (incluyendo las variables dummy) contando con, al menos, 10 sujetos por variable ${ }^{25}$.

\section{RESULTADOS}

Fueron entrevistados 451 sujetos. Rechazaron participar otros 36 que no presentaban diferencias con el grupo respecto a su «necesidad objetibable» en salud ni en la utilización del servicio. En la tabla 1 se recogen las características de las personas incluidas en el estudio.

El 25\% de los pacientes habían realizado menos de 7 visitas, el 50\% menos de 13 y el $75 \%$ menos de 20 en el último año. Una tercera parte de la población había realizado más de 17 visitas en este periodo, y fueron catalogados como Utilizadores Frecuentes (UF).

En la tabla 2 se resume el modelo elegido, cuya variable dependiente es el ln del número de consultas anuales, el cual explica un $20,1 \%$ de la varianza. Si introducimos las variables por bloques, la CVRS (EuroQol$5 \mathrm{D}$, utilidades) explica un 5,2\% de la variabilidad, vivir en barrios de rentas altas un 
Tabla 1

Características de los sujetos participantes en el estudio

\begin{tabular}{|c|c|c|c|}
\hline & $\begin{array}{l}\text { Porcentajes } \\
\text { sobre el total }\end{array}$ & $\begin{array}{c}\text { Media } \\
\text { (IC 95\%) }\end{array}$ & $\begin{array}{l}\text { Mediana } \\
\text { (rango IC) }\end{array}$ \\
\hline Edad & & $57,3(56,0-58,7)$ & $57,0(45,0-70,0)$ \\
\hline Sexo (varón/mujer) & $36,6 \% / 63,4 \%$ & & \\
\hline Nacionalidad (española/otra) & $89,6 \% / 10,4 \%$ & & \\
\hline Otro aseguramiento (sí/no) & $23,5 \% / 76,5 \%$ & & \\
\hline Enfermedades crónicas (sí/no) & $71,2 \% / 28,8 \%$ & & \\
\hline Ingresos hospitalarios (sí/no) & $20,0 \% / 80,0 \%$ & & \\
\hline EuroQol 5D EVA & & $64,4(62,5-66,4)$ & $50,0(60,0-80,0)$ \\
\hline EuroQol_5D Utilidad & & $0,70(0,67-0,73)$ & $0,83(0,42-0,91)$ \\
\hline \multicolumn{4}{|l|}{ Tiempo en conseguir consulta } \\
\hline En el mismo día & $26,6 \%$ & & \\
\hline Un día & $33,0 \%$ & & \\
\hline Dos días & $19,1 \%$ & & \\
\hline Tres días & $5,1 \%$ & & \\
\hline Más de tres días & $16,2 \%$ & & \\
\hline \multicolumn{4}{|l|}{ Tiempo de espera en consulta } \\
\hline Menos de 15 minutos & $70,5 \%$ & & \\
\hline Entre 16 y 30 minutos & $23,1 \%$ & & \\
\hline Entre 31 y 60 minutos & $5,5 \%$ & & \\
\hline Más de una hora & $0,9 \%$ & & \\
\hline \multicolumn{4}{|l|}{ Nivel de estudios } \\
\hline Analfabetos & $0,9 \%$ & & \\
\hline Sin estudios & $20,4 \%$ & & \\
\hline Estudios primarios & $37,9 \%$ & & \\
\hline Enseñanza secundaria & $25,5 \%$ & & \\
\hline Enseñanza superior & $15,3 \%$ & & \\
\hline \multicolumn{4}{|l|}{ Grupo social } \\
\hline Gerentes, directivos & $12,9 \%$ & & \\
\hline Cargos intermedios & $16,0 \%$ & & \\
\hline Trabajador cualificado no manual & $13,5 \%$ & & \\
\hline Trabajador cualificado manual & $40,8 \%$ & & \\
\hline Trabajador parcialmente cualificado manual & $2,7 \%$ & & \\
\hline Trabajador manual no cualificado & $14.3 \%$ & & \\
\hline Tamaño familiar & & $2,8(2,7-2,9)$ & $3(2-4)$ \\
\hline Renta individual $(€)$ & & $\begin{array}{c}1288,8 \\
(1200,0-1377,6) \\
\end{array}$ & $\begin{array}{c}966,6 \\
(682,1-1591,5) \\
\end{array}$ \\
\hline $\mathrm{N}^{\circ}$ visitas al médico / año & & $15,3(14,3-16,4)$ & $13(7-20)$ \\
\hline
\end{tabular}

IC 95\%: Intervalo de confianza del 95\%; Rango IC: rango intercuartílico (percentil 25-percentil 75); EuroQol 5D: Cuestionario EuroQol 5D que consta de Escala Visual Analógica (EVA) y Utilidades 
Tabla 2

Modelo explicativo para el número de consultas anual

\begin{tabular}{|l|c|c|c|c|c|c|}
\hline & \multicolumn{2}{|c|}{$\begin{array}{c}\text { Coeficientes no } \\
\text { estandarizados }\end{array}$} & Sig. & \multicolumn{2}{c|}{$\begin{array}{c}\text { Intervalo de confianza } \\
\text { del 95\% para B }\end{array}$} & FIV \\
\hline & B. & $\begin{array}{c}\text { Robust } \\
\text { D.S. }\end{array}$ & & $\begin{array}{c}\text { Límite } \\
\text { inferior }\end{array}$ & $\begin{array}{c}\text { Límite } \\
\text { superior }\end{array}$ & \\
\hline Constante & 2,352 & 0,235 & $<0,001$ & 1,890 & 2,814 & \\
\hline $\begin{array}{l}\text { Utilidad EuroQol } \\
\text { 5D recodificada }\end{array}$ & $-0,149$ & 0,042 & $<0,001$ & $-0,232$ & $-0,066$ & 1,038 \\
\hline Renta media zona & $-0,192$ & 0,074 & 0,010 & $-0,337$ & $-0,046$ & 1,102 \\
\hline Edad & 0,008 & 0,003 & 0,008 & 0,002 & 0,014 & 1,442 \\
\hline Renta individual & $-9,5 \mathrm{E}-5$ & $-3,8 \mathrm{E}-5$ & 0,014 & $-17,2 \mathrm{E}-5$ & $-1,9 \mathrm{E}-5$ & 1,156 \\
\hline Ingresos & 0,235 & 0,010 & 0,019 & 0,039 & 0,431 & 1,016 \\
\hline $\begin{array}{l}\text { Enfermedades } \\
\text { crónicas }\end{array}$ & 0,418 & 0,105 & $<0,001$ & 0,211 & 0,625 & 1,386 \\
\hline
\end{tabular}

Variable dependiente: In (número de visitas/año).

N 451, F 20,610 p<0,001, R R $^{2} 210$.

Robust DS: estimadores robustos de la Desviación Estándar.

Sig: Significación.

FIV: Factor de Inflación de la Varianza.

$2,4 \%$, la edad añade un $7,4 \%$ de la capacidad explicativa, la renta individual un $1,0 \%$ y los factores relacionados con la «necesidad» (enfermedades crónicas e ingresos hospitalarios) explican un $4,1 \%$ adicional de la variabilidad.

Cada aumento de tercil en la distribución de las utilidades (EuroQol-5D) se asocia con una disminución de 0,149 puntos en el ln del número de consultas. Esto es, cada aumento de tercil en la distribución de las utilidades supone una disminución media del 13,8\% de las consultas anuales $\left(1-\mathrm{e}^{-0,149}\right)$. El hecho de residir en una zona de renta económica en el tercil alto disminuye un $17,5 \%$ de media las consultas anuales. Por cada mil euros que aumenta la renta individual, disminuyen un 9,5\% las consultas anuales de media. El número de consultas aumenta con la edad (un $8,0 \%$ por década). El hecho de haber sufrido enfermedades crónicas o ingresos hospitalarios en el último año aumenta el número medio de consultas $(51,9$ y $26,5 \%$ respectivamente).

El poseer otro tipo de aseguramiento (elemento facilitador individual) y la renta de la zona (factor ambiental) son dos variables muy correlacionadas, por lo que no pueden ser incluidas en el mismo modelo. Si sustituimos en el modelo la renta de la zona por el poseer otro aseguramiento (datos no mostrados) la capacidad explicativa apenas se modificaría y esta variable se asociaría con una disminución del 17,5\% en las consultas anuales medias, con una significación marginal (IC 95\%: - 29,8\%- 1,5\%, $\mathrm{p}=0,072$ ).

Otros elementos predisponentes como el nivel de estudios, el tamaño familiar o la ocupación, o facilitadores como las medidas de accesibilidad, no explicaban las diferencias en el uso del servicio. 
Tabla 3

Modelo explicativo sobre variables asociadas con la pertenencia al tercil superior del número de consultas

\begin{tabular}{|l|c|c|c|c|cc|}
\hline & B & Robust & Sig. & Exp(B) & \multicolumn{2}{|c|}{$\begin{array}{c}\text { Intervalo de confianza } \\
\text { del 95\% para B }\end{array}$} \\
\cline { 5 - 7 } & & & $\begin{array}{c}\text { Límite } \\
\text { inferior }\end{array}$ & $\begin{array}{c}\text { Límite } \\
\text { superior }\end{array}$ \\
\hline Constante & $-1,056$ & 0,631 & 0,094 & & & \\
\hline $\begin{array}{l}\text { Utilidad EuroQol 5D } \\
\text { recodificada }\end{array}$ & & & & & & \\
\hline $2^{\text {a }}$ Tercil vs 1 & & & & & 0,358 & 1,136 \\
\hline $3^{\text {er }}$ tercil vs 1 & $-0,449$ & 0,295 & 0,127 & 0,638 & 0,333 & 0,884 \\
\hline Renta media zona & $-0,611$ & 0,249 & 0,014 & 0,452 & 0,383 & 0,940 \\
\hline Edad & $-0,511$ & 0,229 & 0,026 & 0,599 & 1,004 & 1,039 \\
\hline Renta individual & 0,021 & 0,009 & 0,017 & 1,021 & 0,999 & 1,000 \\
\hline Ingresos & $-4,1 \mathrm{E}-4$ & $1,5 \mathrm{E}-4$ & 0,007 & 0,999 & 1,582 & 4,517 \\
\hline Enfermedades crónicas & 0,653 & 0,302 & 0,031 & 1,921 & 1,062 & 3,477 \\
\hline
\end{tabular}

Variable dependiente: 18 o más visitas/año.

$\mathrm{N} 451, \mathrm{Chi}^{2}$ 67,480 p<0,001, pseudo $\mathrm{R}^{2} 0,132$.

Robust DS: estimadores robustos de la Desviación Estándar.

Sig: Significación.

El modelo logístico se muestra en la tabla 3. La CVRS es relevante en la clasificación de los posibles UF. Estar en el tercil más alto de la distribución de utilidades del Euroqol 5D (que implica una mejor percepción de la CVRS) disminuye un $54,8 \%$ la probabilidad de ser un UF respecto al tercil inferior, ajustando por el resto de los factores explicativos. También reduce esta probabilidad un $40,1 \%$ vivir en zonas de renta alta. Existe una tendencia a aumentar la probabilidad de ser UF con la edad, un $21,0 \%$ por cada década. Cada aumento de 1000 euros en la renta individual, disminuye un 40, $2 \%$ la probabilidad de ser un UF. Se duplica ampliamente la probabilidad de ser UF si se ha ingresado en el último año, y aumenta un 92,1 esa probabilidad si se padecen enfermedades crónicas. El modelo clasifica correctamente en un 63,6 \% de los casos de UF en el conjunto de la muestra y posee una sensibilidad del $85,4 \%$ (IC95\%: $82,1-88,7 \%$ ) y una especificidad del 52,7\% (IC 95\%: 48,1-57,3\%).

\section{DISCUSIÓN}

La calidad de vida relacionada con la salud (CVRS), un «resultado» del contacto con el sistema sanitario según el «modelo conductual», es un factor explicativo importante de la variabilidad en el número de contactos con el médico de familia, entre la población que consulta en un sistema público de salud. Aporta tanta capacidad explicativa como los factores relativos a la «necesidad objetivable», recogidos en este estudio.

Otros factores relacionados con el entorno o con características individuales también 
muestran su capacidad explicativa en la variabilidad de la utilización de la consulta y en la capacidad para clasificar a los sujetos que hacen una utilización más frecuente de la consulta del médico de familia. Entre los factores relacionados con el entorno destaca la zona de residencia, entre los predisponentes la edad, y entre los facilitadores el nivel de renta. La necesidad objetiva, manifestada por al presencia de enfermedades crónicas e ingresos hospitalarios, como era esperable, en un factor decisivo a la hora de explicar las diferencias en la utilización.

Estos resultados merecen ser comentados desde dos perspectivas implícitas en el propio «modelo conductual», la equidad en el uso y las implicaciones en la planificación de los servicios.

Andersen definía la utilización «equitativa» como aquella cuya variabilidad era explicada sobre todo por factores demográficos y de necesidad. Efectivamente la edad y las variables que relacionamos con la necesidad en salud, explican la variabilidad de la utilización en el caso que presentamos. Pero otras variables que pueden actuar como factores ambientales o individuales «facilitadores» (la zona de residencia y los ingresos individuales), también relacionados con la variabilidad en la utilización, no actúan como limitadores de ésta, sino que la aumentan, poniendo de manifiesto lo que se ha dado en llamar inequidad «pro-pobres» en nuestro medio. Este sesgo o inequidad «pro-pobres» se ha descrito ampliamente en la literatura respecto al uso de la atención primaria en los servicios públicos de salud. Desde nuestro punto de vista, este uso más frecuente de servicios sanitarios, no puede considerarse un fallo del sistema, pues puede ser solo expresión de un desplazamiento de la utilización hacia otros niveles asistenciales, o hacia el entorno privado, por parte de otros estratos económicos, como se ha mostrado en otros trabajos ${ }^{26}$.

No existen otros factores facilitadores que puedan explicar inequidades en el acceso. $\mathrm{La}$ accesibilidad no resultó influyente en la utilización del servicio estudiado. La distancia al centro sanitario y el tiempo de espera no suponen en nuestro medio una barrera a la utilización de la atención primaria, a diferencia de lo sugerido anteriormente. Los centros de salud se sitúan a una distancia perfectamente accesible para la gran mayoría de la población ${ }^{27}$. El $60 \%$ de los pacientes tenían cita en el mismo día o el siguiente, y el 93,6\% esperaron menos de 30 minutos a ser atendidos.

La CVRS es una característica individual cuya evaluación tiene importancia tanto para la asistencia individual, como para la planificación de servicios, pues es un resultado del contacto con el sistema sanitario, y también tiene una clara relación con la utilización de la consulta del médico de familia, como se muestra en los dos modelos propuestos. Dentro de sistemas públicos, una peor percepción de la CVRS se había mostrado como predictora de un mayor uso de la consulta del médico de familia en determinadas patologías crónicas, pero parecía que la CVRS no explicaba consistentemente la utilización de los servicios públicos de salud. Los resultados de este trabajo apoyan su uso en el campo de la planificación, pues conocer la percepción de la CVRS puede servir para identificar pacientes que hacen una utilización más frecuente de la consulta del médico de familia.

Las implicaciones de este estudio en la planificación de servicios han de valorarse desde el propio modelo. El uso de recursos sanitarios crece de manera continuada, y en nuestro país, los pacientes que sólo tiene seguro público acuden tres veces más al médico de familia que al resto de especialidades en su conjunto, tendencia que se invierte entre los seguros privados ${ }^{28}$. En la Comunidad de Madrid el 62,9\% de sus habitantes consultaron en Atención Primaria en $2005^{29}$. El $50 \%$ de los encuestados ha contactado, al menos, 13 veces a su médico de familia en el último año. En diversos estu- 
dios sobre utilización de servicios, se establece un punto de corte para definir la utilización frecuente o hiperfrecuentación entre 9 y 12 visitas/año ${ }^{30}$. Estos trabajos utilizan datos poblacionales, mientras que nosotros solo utilizamos datos de usuarios efectivos del servicio. Por otra parte, no se puede identificar el uso inadecuado de servicios por el número de veces que se contacta con el sistema, sin entrar a valorar la adecuación de cada visita, lo que lleva a nuevas propuestas en la definición de pacientes hiperfrecuentadores en función de su situación clínica ${ }^{31}$. Pero nuestro objetivo no era definir el umbral de la utilización normal de recursos, sino estudiar la variabilidad en el uso bajo el «modelo conductual».

Este trabajo presenta algunas limitaciones. Aunque el «modelo conductual» puede usarse como explicativo o predictivo de la utilización, se ha diseñado un estudio transversal, que nos impide establecer relaciones de causalidad y efectuar predicciones. Por otra parte solo se tiene información sobre los sujetos que acuden a consulta, lo que excluye a una parte significativa de la población. Tampoco podemos intentar estimar la utilización en nuestro medio de forma precisa, pues la muestra solo es representativa de los centros que fueron seleccionados por dos características que creímos relevantes, la renta de la zona y el hecho de la ruralidad. $\mathrm{La}$ utilización también puede estar sobrestimada por haber muestreado los listados de asistencia a consultas, lo que aumenta la probabilidad de inclusión de los sujetos que más consultan. Estas son limitaciones a la validez externa de los resultados, pero que no ponen en cuestión la validez del diseño para estudiar la variabilidad de la utilización. Otra limitación es la capacidad explicativa del modelo, alrededor del 20\%. Aunque no es una cifra desalentadora para un modelo generado con microdatos, somos conscientes de que las diferencias en la utilización en trabajos hechos en nuestro país han sido explicadas en un rango que oscila entre el 40 y el $50 \%{ }^{7,32}$. Estas diferencias pueden venir explicadas por varias características, como el diseño prospectivo en uno de los casos, la inclusión de variables diseñadas para tener un alto poder discriminativo como los Ambulatory care groups (ACGs), disponibles para los gestores pero no para el médico asistencial y la inclusión de otras variables predisponentes individuales como el apoyo social. Entendemos que ajustes superiores al $50 \%$ de capacidad explicativa fuera de diseños ecológicos, pueden deberse más a debilidades que a verdaderas fortalezas en el diseño de los estudios.

Como aspectos valorables del presente trabajo mencionamos la fidelidad del registro de la utilización, que evita los sesgos de memoria que supone el preguntar estos datos al paciente ${ }^{33}$, el obtener todos los datos de forma individual y la medición de la variable de estudio, de una herramienta de medida ampliamente validada y contrastada, el EuroQol-5D.

Los resultados expuestos tienen trascendencia en el contexto en el que se producen. Aunque no hay evidencias de la eficacia de intervenciones para disminuir la sobreutilización en atención primaria ${ }^{34}$, puede ser útil para el médico valorar la percepción del paciente respecto su propio estado de salud a través de la CVRS, pues le ayudará a identificar pacientes, a priori, más proclives a esta circunstancia, para entenderla y no favorecerla. El «modelo conductual» ofrece soluciones para valorar la equidad de la utilización pero también su efectividad y su eficiencia que será mayor cuanto más incremente la relación entre el estado de salud o la satisfacción y el uso de servicios. Este estudio no está diseñado para dar respuesta a ese tipo de preguntas, pero si permite identificar circunstancias asociadas a una mayor utilización de la consulta del médico de familia, lo que tiene interés a la hora de asignar recursos.

En conclusión, parece establecido que la CVRS además de ser un resultado del con- 
tacto con el sistema sanitario, se asocia de manera independiente con la variabilidad en la utilización de la consulta del médico de familia, una vez ajustado el efecto de la necesidad en salud, como se propone en el «modelo conductual». Los factores económicos tanto ambientales, como personales también modulan esta relación y no hay factores facilitadores aparentes que justifiquen inequidades en la capacidad de acceder a este servicio para los colectivos menos favorecidos económicamente.

Futuros trabajos podrán determinar si el «modelo conductual», tiene aplicabilidad como modelo predictivo en el contexto de la consulta del médico de familia en nuestro medio.

\section{BIBLIOGRAFÍA}

1. Smits FT, Brouwer HJ, ter Riet G, et al. Epidemiology of frequent attenders: a 3-year historic cohort study comparing attendance, morbidity and prescriptions of one-year and persistent frequent attenders. BMC Public Health. 2009; 9:36.

2. Andersen RM. Revisiting the behavioral model and access to medical care: does it matter? J Health Soc Behav. 1995;36:1-10.

3. Andersen RM. National Health Surveys and the Behavioral Model of Health Services Use. Med Care. 2008; 46: 647-53.

4. Arcury TA, Gesler WM, Preisser JS, et al. The effects of geography and spatial behavior on health care utilization among the residents of a rural region. Health Serv Res. 2005; 40: 135-55.

5. Phillips KA, Morrison KR, Andersen R, et al. Understanding the context of healthcare utilization: assessing environmental and provider-related variables in the behavioral model of utilization. Health Serv Res. 1998;33: 571-96.

6. Vedsted P, Olesen F. Social environment and frequent attendance in Danish general practice. Br J Gen Pract. 2005;55:510-5.

7. Bellón JA, Delgado-Sánchez A, de Dios Luna J, et al. Patient psychosocial factors and primary care consultation: a cohort study. Fam Pract. 2007; 24: 562-9.
8. Pérez MA, Moreno VM, Puerta DR, et al. Factores socioeconómicos y frecuentación en las consultas de medicina de familia de la red sanitaria pública madrileña. Gac Sanit. 2007;21: 219-26.

9. Van Doorslaer E, Masseria C, Koolman X: Inequalities in access to medical care by income in developed countries. CMAJ. 2006;174:177-83.

10. Carr-Hill RA, Rice N, Roland M. Socioeconomic determinants of rates of consultation in general practice based on fourth national morbidity survey of general practices. BMJ. 1996; 312: 1008-12.

11. Health Utilisation Research Alliance (HURA). Ethnicity, socioeconomic deprivation and consultation rates in New Zealand general practice. J Health Serv Res Policy. 2006;11:141-9.

12. Regidor E, Martínez D, Calle ME, et al. Socioeconomic patterns in the use of public and private health services and equity in health care. BMC Health Serv Res. 2008;8:183.

13. Lostao L, Regidor E, Calle ME, et al. Evolución de las diferencias socioeconómicas en la utilización y accesibilidad de los servicios sanitarios en España entre 1987 y 1995-97. Rev Esp Salud Pública. 2001;75:115-28.

14. Regidor E, Martínez D, Astasio P, et al. Asociación de los ingresos económicos con la utilización y la accesibilidad de los servicios sanitarios en España al inicio del siglo XXI. Gac Sanit. 2006; 20: 352-9.

15. Mello MM, Stearns SC, Norton EC. Do Medicare HMOs still reduce health services use after controlling for selection bias? Health Economics. 2002;11:323-40.

16. Knox SA, Britt H. The contribution of demographic and morbidity factors to self-reported visit frequency of patients: a cross-sectional study of general practice patients in Australia. BMC Family Practice. 2004, 5:17.

17. León-Muñoz LM, López-García E, Graciani A, et al. Functional status and use of health care services: Longitudinal study on the older adult population in Spain. Maturitas. 2007; 58:377-86.

18. Bowen ME, González HM. Racial/ethnic differences in the relationship between the use of health care services and functional disability: the health and retirement study (1992-2004). Gerontologist. 2008;48:659-67.

19. Pappa E, Niakas D. Assessment of health care needs and utilization in a mixed public-private sys- 
tem: the case of the Athens area BMC Health Serv Res. 2006;6:146.

20. Parkerson GR Jr, Harrell FE Jr, Hammond WE, et al. Characteristics of adult primary care patients as predictors of future health services charges. Med Care. 2001; 39:1170-81.

21. Rosemann T, Joos S, Szecsenyi J, et al. Health service utilization patterns of primary care patients with osteoarthritis. BMC Health Serv Res. 2007; 7:169.

22. Von Lengerke T, John J; KORA Study Group. Excess use of general practitioners by obese adults: does health-related quality of life account for the association? Psychol Health Med. 2007;12: 53644.

23. Jordan K, Ong BN, Croft P. Previous consultation and self reported health status as predictors of future demand for primary care. J Epidemiol Commun Health. 2003;57: 109- 13 .

24. Domingo Salvany A, Marcos Alonso J. Propuesta de un indicador de clase social basado en la ocupación. Gac Sanit. 1989; 3: 320-6.

25. Ortega Calvo M, Cayuela Domínguez A. Regresión logística no condicionada y tamaño de muestra: una revisión bibliográfica. Rev Esp Salud Publica. 2002; 76: 85-93.

26. Fusté J, Séculi E, Brugulat $\mathrm{P}$, Medina A, Juncà $\mathrm{S}$. Población con cobertura pública o doble cobertura de aseguramiento sanitario. ¿Cuál es la diferencia? Gac Sanit. 2005; 19:15-21.

27. Comunidad de Madrid. Isocronas sanitarias. Estudio de la accesibilidad geográfica a los recursos de la red sanitaria pública en la Comunidad de Madrid. Madrid: Consejería de Salud; 1992.

28. Rodríguez M, Stoyanova A. The effect of private insurance access on the choice of GP/specialist and public/private provider in Spain. Health Econ. 2004; 13: 689-703.

29. Dirección General de Salud Pública y Alimentación. Informe 2007 del estado de salud de la población de la comunidad de Madrid. Disponible en: http://www.madrid.org/cs/Satellite?pagename=Po rtalSalud/Page/PTSA_home. [citado el 1 de octubre de 2009]

30. Vedsted P, Christensen MB. Frequent attenders in general practice care: A literature review with special reference to methodological considerations. Public Health. 2005; 119: 118-137.

31. Luciano Devis JV, Serrano Blanco A, Grupo DASMAP. Los hiperfrecuentadores en atención primaria: perfil sociodemográfico, características clínicas y propuesta de una nueva definición. Aten Primaria. 2009; 40: 631-2.

32. Bolaños-Carmona V, Ocaña-Riola R, PradosTorres A, et al. Variations in health services utilization by primary care patients. Health Serv Manag Res. 2002; 15: 116-25.

33. Bellón JA, Lardelli P, Luna JD, et al. Validity of self reported utilisation of primary health care services in an urban population in Spain. J Epidemiol Community Health. 2000; 54: 544-51.

34. Smits FT, Wittkampf KA, Schene AH, et al. Interventions on frequent attenders in primary care. A systematic literature review. Scand J Prim Health Care. 2008;26:111-6. 